\title{
Postural and Anticonvulsant Effects of Inhibition of the Rat Subthalamic Nucleus
}

\author{
David Dybdal and Karen Gale \\ Interdisciplinary Program in Neuroscience and Department of Pharmacology, Georgetown University Medical Center, \\ Washington, DC 20007
}

The subthalamic nucleus (STN) plays a crucial role as a regulator of basal ganglia outflow by providing excitatory glutamatergic input into the two output nuclei of the basal ganglia, substantia nigra pars reticulata (SNpr), and entopeduncular nucleus. This study examined the effects of suppressing activity in the STN of the awake, behaving rat. Specifically, we evaluated the effects of unilateral and bilateral focal inhibition of STN on posture, locomotion, and susceptibility to limbic motor seizures.

Unilateral microinjection of a $\mathrm{GABA}_{\mathrm{A}}$ receptor agonist (muscimol, $200 \mathrm{pmol}$ ) into STN produced a site-dependent contralaterally directed postural asymmetry without locomotor activation. This effect differed from responses produced by the same dose of muscimol placed into SNpr, which included locomotor activation in addition to contralaterally directed postural asymmetry. Locomotor activation and postural asymmetry were obtained also after blockade of glutamate transmission in SNpr by the unilateral application of kynurenate (100 nmol). Our observation that STN inhibition did not induce the locomotor activation characteristic of SNpr inhibition suggests that there are glutamatergic inputs to SNpr, other than those from STN, that are responsible for controlling locomotion.

Bilateral, but not unilateral, injection of muscimol (200 pmol) into STN protected against limbic motor seizures evoked either by intravenous bicuculline or by focal application of bicuculline into anterior piriform cortex (area tempestas). These results demonstrate that focal inhibition of STN reproduces the postural asymmetry and anticonvulsant actions that are obtained with the inhibition of SNpr. This provides behavioral support for the concept that STN contributes a crucial tonic excitatory (glutamatergic) drive to the rat SNpr.

Key words: subthalamic nucleus; substantia nigra pars reticulata; GABA; muscimol; seizures; posture
The basal ganglia are composed of the caudate, putamen, subthalamic nucleus (STN), globus pallidus (external and internal segments), and substantia nigra (pars compacta and pars reticulata) (Côté and Crutcher, 1991; Thach, 1999). The pars reticulata of substantia nigra (SNpr) together with the closely related internal segment of the globus pallidus (also known as entopeduncular nucleus, EPN) constitute the output nuclei of the basal ganglia. Thus, SNpr plays a pivotal role in the control of posture and movement, as evidenced by its involvement in Parkinson's disease, dystonias, and dyskinesias (for review, see Scheel-Kruger, 1986). Moreover, evidence from the rat indicates that SNpr outputs can regulate cerebral excitability and thereby influence susceptibility to many types of epileptic seizures (for review, see Proctor and Gale, 1998).

Extensive studies of the neuropharmacology and functional neuroanatomy of SNpr in the rat have established that (1) $\mathrm{SNpr}$ regulates posture and locomotion. Unilateral intranigral microinjection of $\mathrm{GABA}_{\mathrm{A}}$ agonists (Oberlander et al., 1977; Olpe et al., 1977; Scheel-Kruger et al., 1977; Olianas et al., 1978; Waddington, 1978a,b; Kozlowski and Marshall, 1980; Imperato and DiChiara, 1981; Childs and Gale, 1984) or glutamate antagonists (Dawbarn and Pycock, 1981; St-Pierre and Bedard, 1994; Murer et al., 1996) results in locomotor activation, contralaterally directed circling, and postural asymmetry. Bilateral intranigral application of $\mathrm{GABA}_{\mathrm{A}}$ agonists induces stereotyped hyperactivity (sniffing and gnawing; Scheel-Kruger et al., 1977; Childs and Gale, 1983; Baumeister and Frye, 1984). These effects are mediated via GABAergic projections from SNpr to the deep layers of the superior colliculus and to the pedunculopontine nucleus (Imperato and

\footnotetext{
Received Jan. 28, 2000; revised June 16, 2000; accepted June 16, 2000.

This work was supported by National Institutes of Health Grants NS20576 and F31 MH10812. We give special thanks to D. Burnhill and K. Japikse for their technical support.

Correspondence should be addressed to Dr. Karen Gale, Department of Pharmacology, Georgetown University Medical Center, 3900 Reservoir Road NW, Washington, DC 20007. E-mail: galek@georgetown.edu; Dave_Dybdal@yahoo.com.

Copyright (C) 2000 Society for Neuroscience $0270-6474 / 00 / 206728-06 \$ 15.00 / 0$
}

DiChiara, 1981; Imperato et al., 1981; Childs and Gale, 1983, 1984). (2) SNpr regulates seizure susceptibility and represents a major site of action of the anticonvulsant effect of GABAergic drugs (Proctor and Gale, 1998). Bilateral inhibition of SNpr has been shown to be anticonvulsant in a number of seizure models, including generalized convulsive seizures (Iadarola and Gale, 1982; De Sarro et al., 1984), generalized nonconvulsive seizures (Depaulis et al., 1988; Deransart et al., 1996), and limbic motor seizures (Turski et al., 1986; Maggio and Gale, 1989). These anticonvulsant effects are mediated primarily via GABAergic projections from the SNpr to the deep layers of superior colliculus (Garant and Gale, 1987).

The SNpr in the rat derives a substantial portion of its excitatory afferents from the STN. Electrophysiological studies in vitro and in anesthetized rats (Nakanshi et al., 1987a,b, 1991) and anatomical tracing studies (Kita and Kitai, 1987; Takada et al., 1988) have demonstrated that the rat STN sends excitatory inputs into both SNpr and EPN. Furthermore, in both the rat and cat the projections from STN to SNpr have been characterized as glutamatergic [rat, Robledo and Feger (1990); Brotchie and Crossman (1991); Price et al. (1993); cat, Albin et al. (1989); Rinvik and Otterson (1993)] and subject to inhibition by GABA within STN [rat, Robledo and Feger (1990); cat, Murer and Pazo (1993)]. However, there have been no systematic studies of the influence of STN on both behavior and seizure susceptibility in the awake, freely moving rat.

On the basis of the proposal that STN is an important source of excitatory input into SNpr, we hypothesized that inhibition of STN would reproduce effects obtained with the inhibition of SNpr in the rat. To test this hypothesis, we evaluated whether unilateral GABA-mediated inhibition of STN (by direct application of the GABA $_{\mathrm{A}}$ agonist muscimol) could induce contralaterally directed circling and postural asymmetry and whether bilateral GABAmediated inhibition of STN could induce stereotyped hyperactivity and an increased resistance to seizures.

Preliminary findings have appeared as abstracts (Dybdal et al., 1995; Dybdal and Gale, 1996). 


\section{MATERIALS AND METHODS}

Animals

Adult male Sprague Dawley albino rats weighing 300-350 gm were used. Rats were housed in groups of four to five per cage, in environmentally controlled conditions (12 hr light/dark cycle, ambient temperature $23 \pm$ $1^{\circ} \mathrm{C}$ ), with food and water available ad libitum. All experiments were conducted during the light cycle in awake, freely moving animals and were performed in adherence to protocols approved by the Georgetown University Animal Care and Use Committee.

\section{Implantation of intracerebral guide cannulae}

Rats were prepared surgically $2-3$ d before experimental testing. We stereotaxically implanted chronic intracerebral guide cannulae into anesthetized rats, as previously described (Maggio and Gale, 1989). Cannulae were positioned (see coordinates below) through holes drilled in the skull and were fixed in place with dental acrylic and jeweler's screws. All of the injection coordinates (see below) were based on the atlas of Pellegrino et al. (1973) (with the tooth bar positioned $5 \mathrm{~mm}$ above the ear bar). Cannulae were directed to the STN, SNpr, EPN, the posterior border of the STN, or $2 \mathrm{~mm}$ dorsal to the STN injection site. In animals tested for focally evoked seizures, an additional cannula was directed toward the left area tempestas (AT), an epileptogenic trigger site in the deep anterior piriform cortex from which limbic motor seizures can be evoked (Piredda and Gale, 1985).

\section{Experimental groups}

For experiments evaluating postural asymmetry, unilateral cannulae were placed into STN, SNpr, EPN, posterior STN, or $2 \mathrm{~mm}$ dorsal to the STN injection site. Coordinates for injection sites were as follows.

$S T N$. Cannulae were placed $1.6 \mathrm{~mm}$ posterior to bregma, $2.3 \mathrm{~mm}$ lateral to midline, and $8.0 \mathrm{~mm}$ below dura. Animals were tested with either saline $(n=6)$ or muscimol $(200 \mathrm{pmol}, n=7)$.

SNpr. Cannulae were placed $3.2 \mathrm{~mm}$ posterior to bregma, $2.3 \mathrm{~mm}$ lateral to midline, and $8.0 \mathrm{~mm}$ below dura. Animals were tested with saline $(n=$ $5)$, muscimol ( $200 \mathrm{pmol}, n=4$; or $45 \mathrm{pmol}, n=4)$, or kynurenate $(100$ nmol, $n=7$ ).

$E P N$. Cannulae were placed $0.6 \mathrm{~mm}$ posterior to bregma, $2.3 \mathrm{~mm}$ lateral to midline, and $8.0 \mathrm{~mm}$ below dura. Animals were tested with either saline $(n=4)$ or muscimol $(200 \mathrm{pmol}, n=6)$.

Posterior border of STN. Cannulae were placed $2.4 \mathrm{~mm}$ posterior to bregma, $2.3 \mathrm{~mm}$ lateral to midline, and $8.0 \mathrm{~mm}$ below dura. In these animals $(n=4)$ the behavior resulting from the injection of muscimol (200 pmol) was compared with the baseline behavior of the same animals in the absence of treatment.

Dorsal to STN. Cannulae were directed $2 \mathrm{~mm}$ dorsal to the STN injection site. All coordinates were the same as for STN except that the ventral position was $6.0 \mathrm{~mm}$ below the dura. The behavior resulting from the injection of muscimol ( 200 pmol, $n=4)$ was compared with the baseline behavior of the same animals in the absence of treatment.

For experiments evaluating anticonvulsant effects, bilateral cannulae were directed toward the STN or dorsal to the STN injection site (as specified above). Two types of seizure models were used.

Focally evoked seizures. An implanted cannula was positioned to allow injections into the left AT $(4.0 \mathrm{~mm}$ anterior to bregma, $3.0 \mathrm{~mm}$ lateral to midline, and $6.5 \mathrm{~mm}$ below dura). To verify accurate injection placement and to ensure a balanced assignment of animals to treatment groups, we evaluated baseline seizure responses by injecting bicuculline methiodide in AT $1 \mathrm{~d}$ before the evaluation of STN treatments. Rats that did not exhibit clonic seizures (score of 3 or higher; see Assessment of Motor Manifestations of Seizure Activity below) during the pretest were not used in subsequent experiments; all others were stratified according to seizure severity to yield five groups with equivalent seizure responses. Each group then was assigned randomly to one of five STN treatments: (1) uninjected controls $(n=5)$, (2) bilateral saline-injected controls $(n=4),(3)$ unilateral muscimol (200 pmol, $n=5)$, (4) bilateral muscimol in STN (200 pmol, $n=$ 5 ), or (5) bilateral muscimol $2 \mathrm{~mm}$ dorsal to the STN injection site (200 pmol, $n=4)$. Injections into STN were made $5 \mathrm{~min}$ before injection of bicuculline methiodide ( $240 \mathrm{pmol})$ into AT.

Systemically evoked seizures. Bilateral cannulae were implanted into the STN in eight animals. Using a balanced cross-over design, we pretreated all eight with either saline or muscimol in the STN 15 min before tail vein injection of bicuculline $(0.35 \mathrm{mg} / \mathrm{kg})$. In addition, five animals received bilateral injections of muscimol $0.5-1 \mathrm{~mm}$ dorsal to the STN injection site.

\section{Microinjection}

Injections were performed over a period of $2-7 \mathrm{~min}$ at a rate between 50 and $100 \mathrm{nl} / \mathrm{min}$ into conscious, freely moving animals. Drug solutions were injected via an acute injection cannula (28 gauge) inserted into the chronically implanted guide cannula ( 22 gauge). The injection cannula, which extended $4 \mathrm{~mm}$ beyond the tip of the guide cannula, was connected via polyethylene tubing to a $10 \mu \mathrm{l}$ Hamilton syringe driven by a Sage inf usion pump. Bilateral infusions were administered via two syringes simultaneously driven by the same pump. The internal cannula was left in place for $1 \mathrm{~min}$ after each infusion before removal. Stainless steel stylettes with dust caps were inserted into guides to keep them patent before, and between, injections.

\section{Drug solutions}

Muscimol (Sigma, St. Louis, MO) was dissolved in saline (200 pmol in 220 nl) and injected into STN, SNpr, or EPN. Kynurenic acid (Research Biochemicals, Natick, MA) was dissolved in a small volume of $1 \mathrm{~N} \mathrm{NaOH}$, diluted with deionized water, and titrated to $\mathrm{pH}$ 6.5. Solutions of kynurenic acid $(100-200 \mathrm{nmol}$ in 385-770 $\mathrm{nl})$ were injected into SNpr.

For focal seizure induction, bicuculline methiodide (Sigma; $240 \mathrm{pmol}$ in $240 \mathrm{nl}$ ) was dissolved in saline and injected into the left AT. For induction of seizures by systemic bicuculline the free base of bicuculline (Sigma) was dissolved in a small volume of $0.1 \mathrm{~N} \mathrm{HCl}$ and then diluted with saline to a concentration of $0.5 \mathrm{mg} / \mathrm{ml}$. The $\mathrm{pH}$ was adjusted to 6 with $\mathrm{NaOH}$, and the solution was kept on ice during the experiment. Bicuculline was injected into the tail vein in a dose of $0.35 \mathrm{mg} / \mathrm{kg}$. The dose of bicuculline was one that consistently induced limbic motor seizures.

\section{Assessment of postural and locomotor effects}

Behavior was observed and recorded immediately before, during, and up to $90 \mathrm{~min}$ after microinjection. During this observation the animals were tested in a $10 \times 12$ inch clear plastic cage. Specific behavioral parameters that were quantified included spontaneous circling and tail lift-evoked circling.

Circling was quantified by counting only rotations that were complete $\left(360^{\circ}\right.$; i.e., portions of incomplete rotations were not summed). For tail lift the animal's tail was held loosely above the hindquarters so that the hindfeet were lifted slightly off the floor $(1-2 \mathrm{~cm})$. This initiated a reflexive escape response in which the rat used its forepaws to locomote in a forward direction. The tail lift thereby converted postural asymmetry into a quantifiable circling behavior. Tail lift-evoked circling was summed over periods of 1 min at time points $2,6,7,10,15,20,25,30,35,45,55$, and 90 min after injection. Spontaneous circling in the test cage was counted and summed over $1 \mathrm{~min}$ intervals sampled at time points immediately before the tail lift measurements.

\section{Assessment of motor manifestations of seizure activity}

Convulsions elicited by microinjection into AT. Behavior was observed and recorded immediately before, during, and up to $90 \mathrm{~min}$ after microinjection. Latency, frequency of occurrence, and duration of convulsive episodes were recorded over a $1 \mathrm{hr}$ period after injection. Severity of seizures was scored as previously described (Maggio and Gale, 1989): 0.5, jaw clonus; 1 , myoclonic jerks of contralateral forelimb; 2 , forelimb clonus ( \pm mouth and facial clonus) lasting $5-15 \mathrm{sec}$; 3 , bilateral forelimb clonus lasting $>15 \mathrm{sec} ; 4$, rearing in addition to bilateral forelimb clonus; 5 , rearing and loss of balance in addition to bilateral forelimb clonus.

Convulsions elicited by intravenous bicuculline. Behavior was observed and recorded immediately before, during, and until the complete cessation of seizure activity ( $\sim 5 \mathrm{~min})$. Latency and duration of convulsive episodes were recorded. To provide an index of seizure severity, we used a point system, adapted from Iadarola and Gale (1982). Points (pt) were assigned for each of the following manifestations: facial twitches, $1 \mathrm{pt}$; forelimb clonus, $1 \mathrm{pt}$; rearing, $1 \mathrm{pt}$; spontaneous jerks, $1 \mathrm{pt}$; spontaneous jumps, $2 \mathrm{pt}$; body twist (quarter, one-half, or full), 1,2 , or $3 \mathrm{pt}$, respectively; prolonged hyperactivity, $1 \mathrm{pt}$; prolonged hyper-responsiveness (jerks or jumps on stimulation), 1 or $2 \mathrm{pt}$, respectively. Points were added to give a total cumulative score for each rat. The maximum possible cumulative score for a single seizure test session for one rat was 12 points.

\section{Histology}

Brains were fixed in formalin (10\%) for at least $24 \mathrm{hr}$ and sectioned coronally (50 $\mu \mathrm{m}$ thick) on a freezing microtome; the sections were stained with neutral red. Evaluation of cannula placement was performed without knowledge of the experimental treatment or response. Animals in which the injection sites failed to fall within the nuclei of interest were eliminated from the data analysis for that group.

\section{Statistical analysis}

For two-group comparisons the Mann-Whitney $U$ test was used for nonparametric data (i.e., seizure severity scores), and the Student's $t$ test (two-tailed) was used for parametric data (i.e., circling rate). For studies comparing three or more groups, the Kruskal-Wallis test was used for nonparametric data, and an ANOVA (Fisher's post hoc) was used for parametric data.

\section{RESULTS}

\section{Postural effects}

Muscimol (200 pmol) microinjected unilaterally into the rat STN (Fig. 1) resulted in a postural asymmetry of the body (twisting toward the contralateral side) and contralaterally directed tilting of the head and neck. This was accompanied by little or no spontaneous circling behavior. However, with the addition of external stimulation (i.e., lifting the tail of the animal), contralaterally 


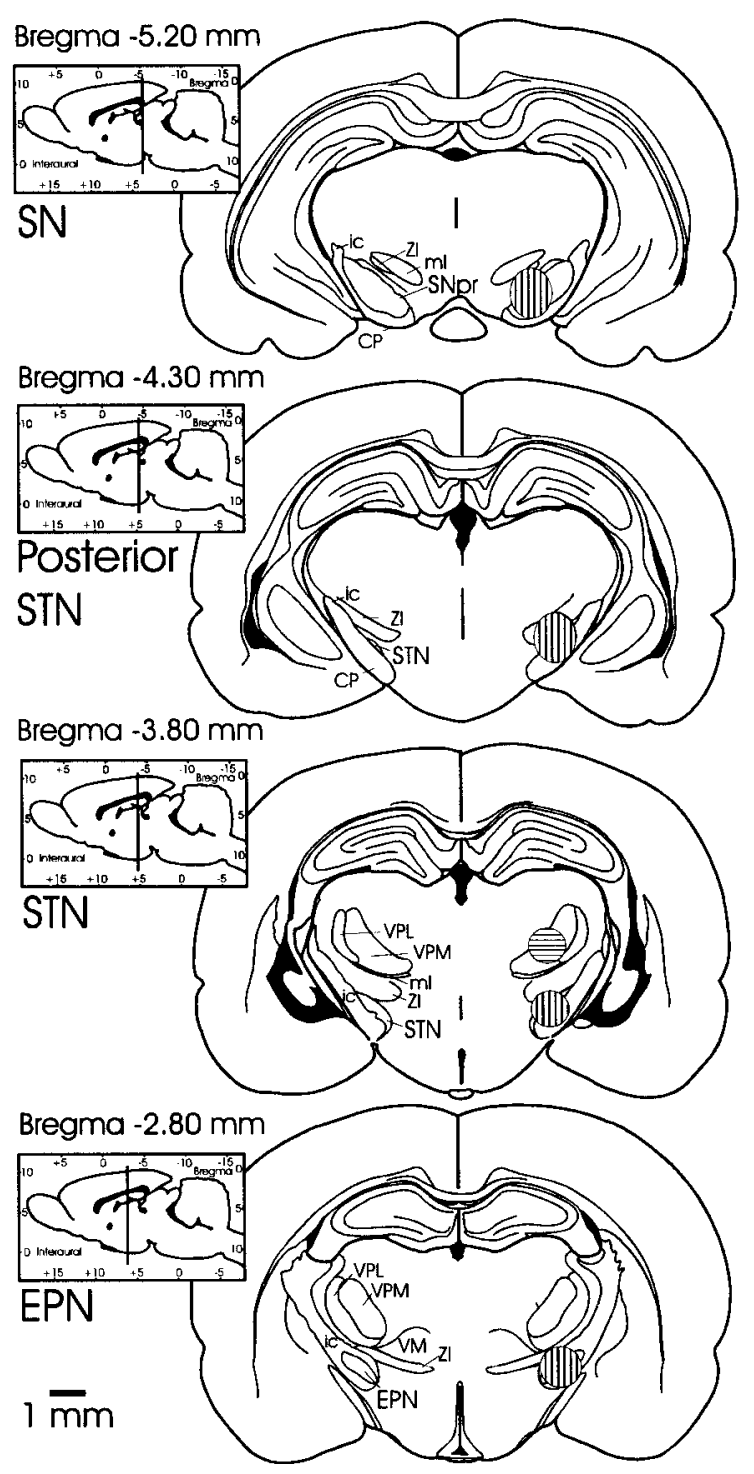

Figure 1. Coronal sections depicting areas of microinfusions into EPN, STN, dorsal to STN, posterior border of STN, and SNpr. Coronal sections for histology were sectioned in the plane corresponding to the atlas of Paxinos and Watson (1997) from which these drawings were derived. Striped areas (shown in one hemisphere only) designate the regions within which injection sites fell. Vertically striped areas designate the regions of EPN, STN, posterior STN, and SNpr injection sites on respective coronal slices. The area of injection sites located dorsal to STN is shown on the same coronal slice as STN and is designated by the horizontally striped area. $C P$, Cerebral peduncle; $E P N$, entopeduncular nucleus; $i c$, internal capsule; $m l$, medial lemniscus; $S N p r$, substantia nigra pars reticulata; $S T N$, subthalamic nucleus; $V M$, ventromedial nucleus of the thalamus; $V P L$, ventral posterolateral thalamic nucleus; $V P M$, ventral posteromedial thalamic nucleus; $Z I$, zona incerta.

directed circling behavior was evoked in the presence of unilateral muscimol in STN (Table 1). In no case was ipsilaterally directed circling observed after unilateral muscimol in STN, either in the presence or absence of tail lift. Rats receiving saline unilaterally in STN showed neither postural asymmetry nor significant circling behavior in either direction, with or without tail lift. The circling evoked by tail lift in the presence of unilateral muscimol in STN appeared with a rapid onset after injection, peaked between 10 and $20 \mathrm{~min}$, and returned to baseline by $90 \mathrm{~min}$ (Fig. 2).

When injections of muscimol $(200 \mathrm{pmol})$ were placed $2 \mathrm{~mm}$ dorsal to the STN injection site (see Fig. 1), no significant postural asymmetry or circling behavior in either direction, in the presence or in the absence of tail lift, was observed (Fig. 3). Muscimol (200 pmol) injected into the EPN (see Fig. 1) resulted in behavior that was similar to that elicited from STN (i.e., contralaterally directed postural asymmetry, with circling behavior seen only in the presence of tail lift; Fig. 3). Injections of muscimol (200 pmol; see Fig. 1) at the posterior border of STN (adjacent to SNpr) resulted in a postural asymmetry with a tail lift-evoked circling rate that was significantly less $(p<0.05)$ than that obtained with injections into STN (Fig. 3).

To allow for a direct comparison of the effects of muscimol in STN with the effects of inhibition in SNpr, we also evaluated the effects of muscimol (200 pmol) and a glutamate antagonist, kynurenate $(100 \mathrm{nmol})$, in SNpr. In contrast to the behavior obtained with muscimol in STN, muscimol (200 or 45 pmol) injected into the center of SNpr resulted in a high rate of contralaterally directed circling in both the presence and absence of tail lift stimulation (Table 1; Fig. 3); this effect confirms well established findings (Oberlander et al., 1977; Olpe et al., 1977; Scheel-Kruger et al., 1977; Olianas et al., 1978; Waddington, 1978a; Kozlowski and Marshall, 1980; Imperato and DiChiara, 1981; Childs and Gale, 1984). Similarly, in agreement with previous studies that have shown contralaterally directed circling with the injection of glutamate antagonists into SNpr (Dawbarn and Pycock, 1981; St-Pierre and Bedard, 1994; Murer et al., 1996), kynurenate injected into the center of the SNpr induced a high rate of contralaterally directed circling both in the presence and absence of tail lift stimulation (Table 1).

Muscimol (200 pmol) microinjected bilaterally into the rat STN evoked a mild stereotyped hyperactive behavior. The predominant rapid onset (within 10 min after injection) effects observed were floor-directed sniffing ( 8 of 10 animals), gnawing (5 of 10), and fine, tremulous head bobs ( 5 of 10). Other effects that occasionally were observed included spontaneous turning (in both directions, but usually one direction predominated; 3 of 10$)$, forelimb movements that appeared as if the limbs were running in place ("running forelimb clonus"; 2 of 10), and rearward locomotion (1 of 10).

\section{Anticonvulsant effects}

Relative to both noninjected and saline-injected controls, bilateral application of muscimol (200 pmol in $220 \mathrm{nl})$ to STN attenuated the severity of limbic motor seizures evoked by the focal application of bicuculline methiodide ( $240 \mathrm{pmol}$ in $240 \mathrm{nl})$ into AT. In contrast, unilateral application of muscimol to STN had no significant effect on seizure severity (Fig. 4). When bilateral muscimol injections were placed $2 \mathrm{~mm}$ dorsal to the STN injection site, no anticonvulsant effect was observed (Fig. 4).

Bilateral muscimol (200 pmol) in STN also decreased the severity of seizures produced by low doses of intravenous bicuculline $(0.35 \mathrm{mg} / \mathrm{kg})$ (Fig. 5). When the bilateral injections were placed in sites dorsal to the STN injection site (these particular injection sites clustered at the ventral border of the dorsal site shown in Fig. 1), the anticonvulsant effect of muscimol was diminished so that the seizure response was not significantly different from control.

\section{DISCUSSION}

Our results demonstrate that the inhibition of STN induces some of the same functional effects as those obtained by the inhibition of SNpr, and provide support for the hypothesis that the STN is an important source of excitatory input into the SNpr in the rat. The postural asymmetry and anticonvulsant effects that we obtained with muscimol application to STN were site-specific and resembled effects that previously have been shown to occur after blockade of excitatory amino acid transmission in SNpr (Dawbarn and Pycock, 1981; De Sarro et al., 1984; Turski et al., 1986; Maggio and Gale, 1989; St-Pierre and Bedard, 1994; Deransart et al., 1996; Murer et al., 1996).

Unilateral application of a GABA agonist into STN resulted in a contralaterally directed postural asymmetry of the body, without locomotor activation (i.e., little or no spontaneous circling). The direction of this asymmetry was the same as that seen after unilateral intranigral GABA agonist application. However, we found that the lack of an increase in spontaneous locomotor activity after unilateral muscimol application in STN was in clear contrast to the 
Table 1. Circling rate after microinjection of muscimol, kynurenate, or saline

\begin{tabular}{|c|c|c|c|c|c|}
\hline & \multicolumn{2}{|c|}{ Tail lift-evoked circles/min } & \multicolumn{2}{|c|}{ Spontaneous circles/min } & \multirow{2}{*}{$\begin{array}{l}\text { Circling ratio: } \\
\text { tail lift/spontaneous } \\
\text { Contra }\end{array}$} \\
\hline & Ipsi & Contra & Ipsi & Contra & \\
\hline \multicolumn{6}{|l|}{ STN treatments } \\
\hline Saline $(n=6)$ & $2.5 \pm 0.4$ & $2.6 \pm 1.2$ & 0 & 0 & \\
\hline Muscimol 200 pmol $(n=7)$ & 0 & $19.9 \pm 2.3^{*}$ & 0 & $1.3 \pm 0.2$ & $14.1 \pm 2.7$ \\
\hline \multicolumn{6}{|l|}{ SNpr treatments } \\
\hline Saline $(n=5)$ & $3.4 \pm 0.3$ & $0.8 \pm 0.5$ & 0 & 0 & \\
\hline Muscimol $200 \operatorname{pmol}(n=4)$ & 0 & $37.8 \pm 6.0^{*}$ & 0 & $13.8 \pm 1.6^{*}$ & $2.7 \pm 0.3^{\#}$ \\
\hline Muscimol 45 pmol $(n=4)$ & 0 & $13.3 \pm 4.0^{*}$ & 0 & $6.7 \pm 2.3^{*}$ & $2.0 \pm 0.2^{\#}$ \\
\hline Kynurenate 100 nmol $(n=7)$ & 0 & $22.7 \pm 2.3^{*}$ & 0 & $6.7 \pm 0.8^{*}$ & $3.4 \pm 0.2^{\#}$ \\
\hline
\end{tabular}

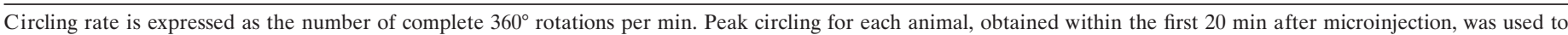

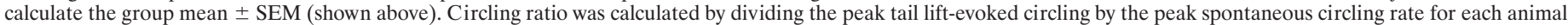
and calculating a group mean ratio \pm SEM.

*Indicates significantly different from respective saline controls.

${ }^{\#}$ Indicates significantly different from ratio of tail lift-evoked to spontaneous circling rates for muscimol in STN ( $p<0.05$, ANOVA and Fisher's post hoc).

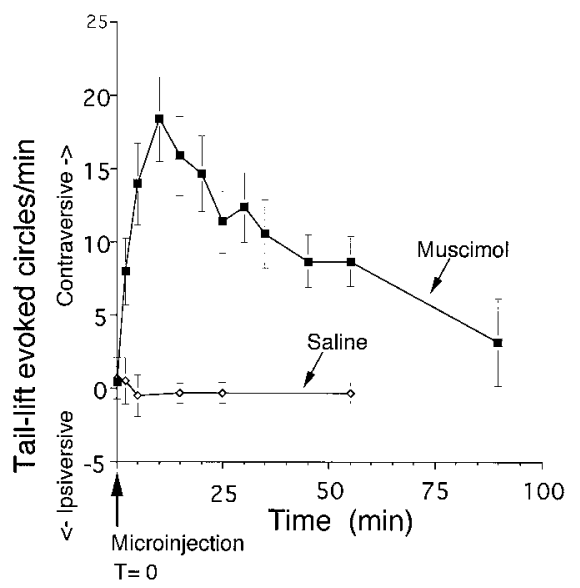

Figure 2. Time course of tail lift-evoked circles. Values represent group means \pm SEM of complete $\left(360^{\circ}\right)$ tail lift-evoked circles/min after muscimol microinjection into STN. Muscimol (200 pmol in $220 \mathrm{nl} ; n=7)$ or saline $(220 \mathrm{nl} ; n=6)$ was injected unilaterally into STN. Circling rates were measured for $1 \mathrm{~min}$ intervals at the time points indicated.

locomotor stimulation that we and others have observed after unilateral application of muscimol in SNpr. The contralaterally directed locomotor stimulation evoked by intranigral muscimol, an effect first reported by Scheel-Kruger et al. (1977), has become a standard endpoint for detecting GABA agonist activity in the rat (Waddington, 1978b; Coward, 1982).

Because the STN is thought to be a major source of glutamatergic input to the SNpr, the lack of locomotor stimulation after STN inhibition raised the question of whether glutamate transmission in SNpr has any influence on locomotor activity. To address this question in our experimental conditions, we injected kynurenate, a broad spectrum glutamate antagonist, unilaterally into the SNpr. Because kynurenate in the SNpr produced locomotor stimulation in addition to postural asymmetry (Table 1), we cannot exclude a role for nigral glutamate in locomotor control. Therefore, the glutamatergic inputs into $\mathrm{SNpr}$ that serve to control locomotor activity are likely to derive from a source other than STN.

One concern encountered in evaluating drug action in STN is that spread of drug to adjacent regions may account for some of the effects that were obtained. Indeed, the central STN injection site was only $1.5-2 \mathrm{~mm}$ anterior to the central SNpr injection site (see Fig. 1). To address the possibility that the postural effects we obtained from the STN injection site were attributable to the diff usion of small amounts of muscimol to SNpr, we injected a low dose of muscimol (45 pmol) directly into SNpr; this dose induced postural asymmetry comparable to that evoked from STN. Consis-

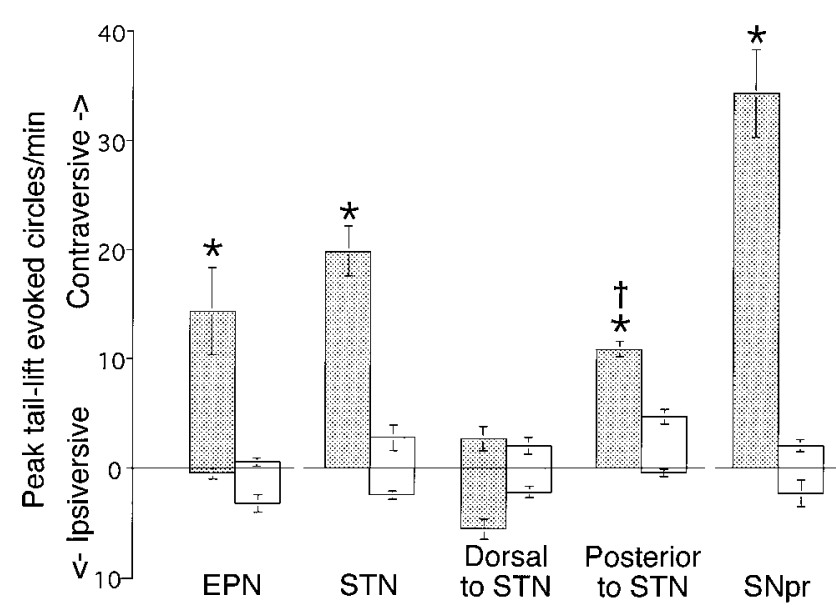

Figure 3. Site specificity of circling behavior evoked by muscimol (200 pmol). Values represent group mean values \pm SEM of peak circling in the presence of tail lift. Peak circling occurred within the first $20 \mathrm{~min}$ after microinjection. Muscimol (200 pmol in $220 \mathrm{nl}$, shaded bars) was injected (in separate groups of rats) into one of five sites. The five sites, in order of appearance on the graph from left to right, are $1 \mathrm{~mm}$ anterior to the STN injection site $(E P N ; n=6)$, STN $(n=7), 2 \mathrm{~mm}$ dorsal to the STN injection site $(n=4), 0.5-1.0 \mathrm{~mm}$ posterior to the STN injection site $(n=4)$, or directly into the SNpr $(n=4)$. Asterisks indicate significantly different from respective controls (clear bars) (Student's $t$ test; $p<0.05$ ); cross indicates significantly different from STN and SN muscimol injection groups (ANOVA and Fisher's post hoc; $p<0.05$ ).

tent with the idea that the postural effects obtained with STN injections were not attributable to diff usion to SNpr, such injections into SNpr produced increased locomotion (i.e., spontaneous circling) in addition to postural asymmetry. Furthermore, the rapid onset and short latency to maximal postural asymmetry (not more than $15 \mathrm{~min}$ ) after muscimol in the STN argues against drug diffusion accounting for this effect. Even at $30 \mathrm{~min}$ after focal injection of muscimol, only trace amounts of muscimol have been detected 1.5-2.5 mm away from the injection site (Sperber et al., 1989). Consistent with this, muscimol injections directed to the border between STN and SNpr $(0.8 \mathrm{~mm}$ caudal to the central STN injection site) produced significantly less effect than injections directly into the center of the STN (see Figs. 1, 3). Finally, injections $2 \mathrm{~mm}$ dorsal to the STN injection site were completely without effect, confirming the high degree of localization of the effects obtained in STN.

Our results are consistent with Scheel-Kruger and Magelund's (1981) description of the behavioral response to muscimol in the STN of Wistar rats. However, because neither quantification of behavior nor time course and site specificity of the drug effects were 


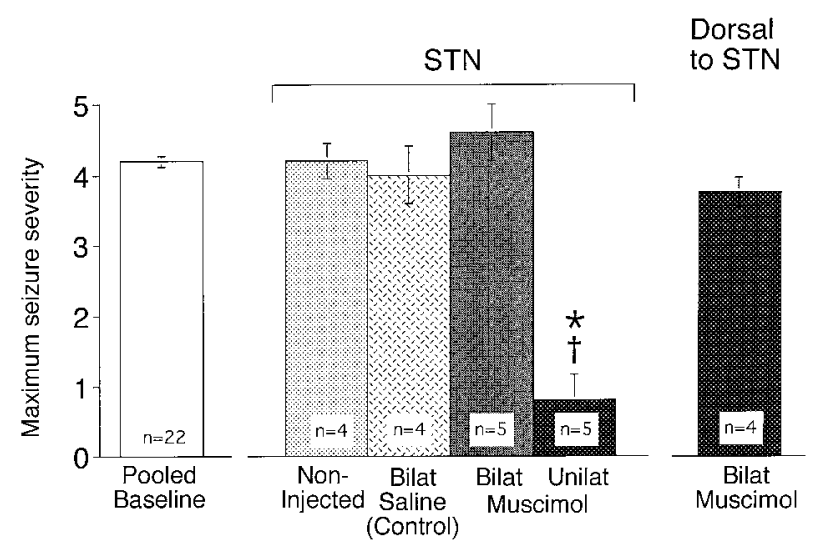

Figure 4. Effect of bilateral infusion of muscimol (200 pmol) into the STN on AT-evoked seizure severity. Seizures were elicited with bicuculline methiodide $(240 \mathrm{pmol}$ in $240 \mathrm{nl})$ injected into the left area tempestas $5 \mathrm{~min}$ after the injection of muscimol into the STN or $2 \mathrm{~mm}$ dorsal to the STN injection site. The STN was bilaterally (Bilat) injected with saline $(220 \mathrm{nl})$ or muscimol (200 pmol in $220 \mathrm{nl}$ on each side) or unilaterally (Unilat, left side) injected with muscimol $(200 \mathrm{pmol}$ in $220 \mathrm{nl})$. The dorsal site was injected bilaterally with saline ( $220 \mathrm{nl})$ or muscimol $(200 \mathrm{pmol}$ in $220 \mathrm{nl}$ on each side). Means of the maximum seizure severity \pm SEM are depicted. Asterisk indicates significant difference with respect to saline control; cross indicates significant difference in comparison to all other groups (KruskalWallis test; $p<0.05)$.

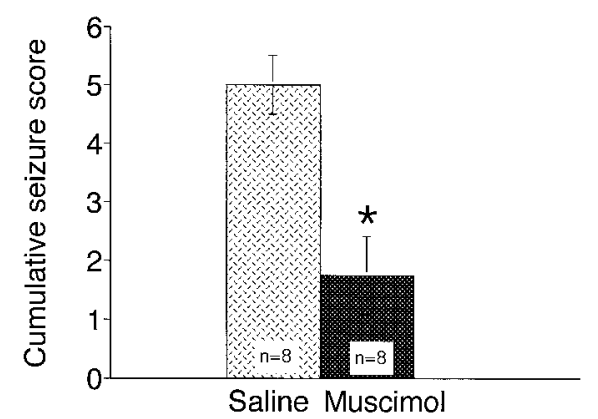

Figure 5. Effect of bilateral inf usion of muscimol $(200 \mathrm{pmol}$ in $220 \mathrm{nl})$ into the STN on the severity of seizures induced by intravenous bicuculline $(0.35$ $\mathrm{mg} / \mathrm{kg}$ ). Bilateral microinjection of muscimol or saline into the STN preceded seizure induction by $15 \mathrm{~min}$. Means of the cumulative seizure score \pm SEM are depicted. Asterisk indicates significant difference with respect to saline-microinjected controls (Mann-Whitney $U ; p<0.05$ ).

presented, it is difficult to compare their results to ours or to assess the extent to which drug action in SNpr may have contributed to their observations. Nevertheless, their descriptions suggest that, as in our studies, muscimol in STN induces clear postural asymmetry. Furthermore, studies in the other species suggest that this relationship also may hold for the cat (Murer and Pazo, 1993).

Bilateral application of muscimol in STN was anticonvulsant in both focal (AT) and systemic (intravenous) models of limbic motor seizures. Unilateral pretreatment with muscimol in STN produced postural asymmetry but had no protective effect in either model of seizure induction. In the AT-evoked seizure model, bilateral STN pretreatment with muscimol protected all animals from clonic seizures (score of 3 or higher). In contrast, no attenuation of seizures occurred after either bilateral saline or unilateral muscimol pretreatment of the STN. In addition, the anticonvulsant effects of muscimol in STN were site-specific, in as much as bilateral muscimol injections, positioned $2 \mathrm{~mm}$ dorsal to the STN, did not result in seizure protection. Bilateral muscimol pretreatment in STN also protected against limbic motor seizures evoked by intravenous bicuculline, indicating that bilateral inhibition of the STN is capable of protecting against both focal and systemic models of seizure induction.

Previous studies in which GABA antagonists (bicuculline and picrotoxin) have been applied to rat STN have described symptoms resembling seizure manifestations after relatively high doses (Scheel-Kruger and Magelund, 1981; Feger et al., 1989). These symptoms include forelimb clonus, myoclonic jerks, jumping, and hypersalivation. We observed similar short-latency responses after unilateral infusion of bicuculline into STN in our animals (our unpublished observations), suggesting that disinhibition of STN may be proconvulsant. The seizure manifestations evoked by disinhibition in the vicinity of STN in the rat have been interpreted as dyskinesias (Crossman et al., 1983; Feger et al., 1989). However, when STN was injected with lower doses of bicuculline or picrotoxin, which are sufficient to produce ipsilaterally directed postural asymmetry without seizure activity, no abnormal movements were manifest (Scheel-Kruger and Magelund, 1981; our unpublished observations). Thus it is likely that STN or an adjacent structure is capable of generating seizure activity upon removal of GABA inhibition in the rat, and that this effect is independent of effects on movement and posture.

We show here that bilateral inhibition of STN protects against focally evoked limbic motor seizures, which are analogous to human complex partial seizures (Piredda and Gale, 1985). This action is comparable to that obtained after bilateral inhibition of SNpr. In addition to protecting against limbic motor seizures (Turski et al., 1986; Maggio et al., 1989), the inhibition of SNpr confers resistance to generalized convulsive seizures (Iadarola and Gale, 1982; De Sarro et al., 1984) and generalized nonconvulsive seizures (Depaulis et al., 1988; Deransart et al., 1996). Therefore, one would expect that bilateral inhibition of the STN, by removal of its excitatory influence on the SNpr, also should be capable of protecting against generalized seizures. Indeed, it recently has been shown that bilateral inhibition of the STN protects against generalized convulsive seizures induced by fluorothyl inhalation (Veliskova et al., 1996), against generalized nonconvulsive seizures in a genetically prone strain of Wistar rat (Deransart et al., 1996), and, more recently, against amygdala-kindled seizures (Deransart et al., 1998).

These findings, taken together, are in agreement with the hypothesis that the STN exerts its influence via the SNpr. However, STN-evoked effects may be mediated via areas other than SNpr. In particular, the STN is known to send glutamatergic projections to the EPN (Robledo and Feger, 1990; Nakanshi et al., 1991), and inhibition of the EPN has been shown to protect against limbic motor seizures (Patel et al., 1986; Hosford and McNamara, 1988). Interestingly, unilateral inhibition of the EPN (with muscimol) induced behavioral effects that were similar to those elicited from STN (i.e., contralaterally directed postural asymmetry, with circling behavior seen only in the presence of tail lift; see Fig. 3). This raises the possibility that the postural influences of STN are mediated in part via excitatory projections to EPN. Clearly, further experiments are necessary to determine the degree to which the influence of STN depends on the SNpr and/or EPN.

Consistent with the proposed crucial role of the STN as a modulator of basal ganglia outputs, this nucleus is associated with the pathophysiology of specific movement disorders. Unilateral lesions involving the STN and adjacent tissue are associated with dyskinesias characterized by hemiballism in the monkey (Whittier and Mettler, 1949; Carpenter et al., 1950; Hamada and DeLong, 1992). Furthermore, lesions of the STN ameliorate the symptoms of experimentally induced parkinsonism in monkeys (Bergman et al., 1990) as well as clinical parkinsonism in humans (Sellal et al., 1992). We recently have shown, however, that the relationship between SN and STN in the monkey (Dybdal et al., 1997) may be very different from the relationship in the rat (as shown here) or cat (Murer and Pazo, 1993); accordingly, there is a need for caution in the extrapolation from rodent models to humans. By developing a greater understanding of the types of behaviors evoked from STN, we can begin to identify the mechanisms by which the STN may exert control over posture and seizure susceptibility. More importantly, when extended into primate studies, an understanding of STN function may suggest novel strategies for the treatment of a variety of neurological disorders such as Parkinson's disease, Huntington's disease, dystonia, and epilepsy. 


\section{REFERENCES}

Albin RL, Aldridge W, Young AB, Gilman S (1989) Feline subthalamic nucleus neurons contain glutamate-like but not GABA-like or glycinelike immunoreactivity. Brain Res 491:185-188.

Baumeister AA, Frye GD (1984) Self-injurious behavior in rats produced by intranigral microinjection of GABA agonists. Pharmacol Biochem Behav 21:89-95.

Bergman H, Wichman T, DeLong MR (1990) Reversal of experimental parkinsonism by lesions of the subthalamic nucleus. Science 249:1436-1438.

Brotchie JM, Crossman AR (1991) D- $\left[{ }^{3} \mathrm{H}\right]$ aspartate and $\left[{ }^{14} \mathrm{C}\right] \mathrm{GABA}$ up take in the basal ganglia of rats following lesions in the subthalamic region suggest a role for excitatory amino acid but not GABA-mediated transmission in subthalamic nucleus efferents. Exp Neurol 113:171-181.

Carpenter MB, Whittier JR, Mettler FA (1950) Analysis of choreoid hyperkinesia in the rhesus monkey: surgical and pharmacological analysis of hyperkinesia resulting from lesions in the subthalamic nucleus of Luys. J Comp Neurol 92:293-332.

Childs JA, Gale K (1983) Evidence that the nigrotegmental GABAergic projection mediates stereotypy induced by apomorphine and intranigral muscimol. Life Sci 33:1007-1010.

Childs JA, Gale K (1984) Circling behavior elicited from the pedunculopontine nucleus: evidence for the involvement of hindbrain GABAergic projections. Brain Res 304:387-391.

Côté L, Crutcher MD (1991) The basal ganglia. In: Principles of neural science, 3rd Ed (Kandel ER, Schwartz JS, Jessel TM, eds), pp 647-659. New York: Elsevier.

Coward DM (1982) Nigral actions of GABA agonists are enhanced by chronic fluphenazine and differentiated by concomitant flurazepam. Psychopharmacology (Berl) 76:294-298.

Crossman AR, Latham A, Longman DA, Patel S, Slater P (1983) Motor dysf unction induced by application of GABA antagonists to subthalamic nucleus of rat brain. J Physiol (Lond) 334:85P.

Dawbarn D, Pycock CJ (1981) Motor effects following application of putative excitatory amino acid antagonists to the region of the mesencephalic dopamine cell bodies in the rat. Naunyn Schmiedebergs Arch Pharmacol 318:100-104.

Depaulis A, Vergnes M, Marescaux C, Lannes B, Warter JM (1988) Evidence that activation of GABA receptors in the substantia nigra suppresses spontaneous spike-and-wave discharges in the rat. Brain Res 448:20-29.

Deransart C, Marescaux C, Depaulis A (1996) Involvement of nigral glutamatergic inputs in the control of seizures in a genetic model of absence epilepsy in the rat. Neuroscience 71:721-728.

Deransart C, Le BT, Marescaux C, Depaulis A (1998) Role of the subthalamo-nigral input in the control of amygdala-kindled seizures in the rat. Brain Res 807:78-83.

De Sarro G, Meldrum BS, Reavill C (1984) Anticonvulsant action of 2-amino-phosphonoheptanoic acid in the substantia nigra. Eur J Pharmacol 106:175-179

Dybdal D, Gale K (1996) Anticonvulsant effect of focal inhibition of the subthalamic nucleus. Soc Neurosci Abstr 22:897.

Dybdal D, Japikse K, Burnhill D, Gale K (1995) Behavioral effect of focal inhibition of the subthalamic nucleus. Soc Neurosci Abstr 21:2079.

Dybdal D, Dubach M, Vaurio R, Estabrooke I, Heath A, Gale K (1997) Focal inhibition of primate substantia nigra: a new model for human dyskinetic and dystonic syndromes. Soc Neurosci Abstr 23:1963.

Feger J, Vezole I, Renwart N, Robledo P (1989) The rat subthalamic nucleus: electrophysiological and behavioral data. In: Current problems in neurology: neural mechanisms in disorders of movement, Vol 9 (Crossman AR, Sambrook MA, eds), pp 37-43. London: Libbey.

Garant DS, Gale K (1987) Substantia nigra-mediated anticonvulsant actions: role of nigral output pathways. Exp Neurol 97:143-159.

Hamada I, DeLong MR (1992) Excitotoxic acid lesions of the primate subthalamic nucleus result in transient dyskinesias of the contralateral limbs. J Neurophysiol 68:1850-1858.

Hosford DA, McNamara JO (1988) Microinjection of muscimol into entopeduncular nucleus suppresses pilocarpine but not maximal electroshock seizures in rats. Brain Res 462:205-210.

Iadarola MJ, Gale K (1982) Substantia nigra: site of anticonvulsant activity mediated by gamma-aminobutyric acid. Science 218:1237-1240.

Imperato A, DiChiara G (1981) Behavioral effects of GABA agonists and antagonists infused in the mesencephalic reticular formation-deep layers of the superior colliculus. Brain Res 224:185-194.

Imperato A, Porceddu ML, Morelli M, Faa G, DiChiara G (1981) Role of dorsal mesencephalic reticular formation and deep layers of superior colliculus as output stations for turning behavior elicited from the substantia nigra pars reticulata. Brain Res 216:437-443.

Kita H, Kitai ST (1987) Efferent projections of the subthalamic nucleus in the rat: light and electron microscopic analysis with the PHA-L method. J Comp Neurol 260:435-452.

Kozlowski MR, Marshall JF (1980) Rotation induced by intranigral injections of GABA agonists and antagonists: zone-specific effects. Pharmacol Biochem Behav 13:561-567.

Maggio R, Gale K (1989) Seizures evoked from area tempestas are subject to control by GABA and glutamate receptors in substantia nigra. Exp Neurol 105:184-188.

Murer G, Pazo JH (1993) Circling behavior induced by activation of $\mathrm{GABA}_{\mathrm{A}}$ receptors in the subthalamic nucleus. NeuroReport 4:1219-1222.
Murer G, Tseng K, Sinay V, Penalva R, Armando I, Pazo JH (1996) Turning behavior induced by injections of glutamate receptor antagonists into the substantia nigra of the rat. Synapse 24:147-155.

Nakanshi H, Kita H, Kitai ST (1987a) Intracellular study of rat substantia nigra pars reticulata neurons in an in vitro slice preparation: electrical membrane properties and response characteristics to subthalamic stimulation. Brain Res 437:35-44.

Nakanshi H, Kita H, Kitai ST (1987b) Electrical membrane properties of rat subthalamic nucleus neurons in an in vitro slice preparation. Brain Res 437:45-55.

Nakanshi H, Kita H, Kitai ST (1991) Intracellular study of rat entopeduncular nucleus neurons in an in vitro slice preparation: response to subthalamic stimulation. Brain Res 549:285-291.

Oberlander C, Dumont C, Boisser JR (1977) Rotational behavior after unilateral intranigral injection of muscimol in rats. Eur $\mathrm{J}$ Pharmacol 43:389-390.

Olianas MC, De Montis GM, Mulas G, Tagliamonte A (1978) The striatal dopaminergic function is mediated by the inhibition of a nigral, nondopaminergic neuronal system via a striatonigral GABAergic pathway. Eur J Pharmacol 49:233-241.

Olpe HR, Schellenberg H, Koella WP (1977) Rotational behavior induced in rats by intranigral application of GABA-related drugs and GABA antagonists. Eur J Pharmacol 45:291-294.

Patel S, Millan M, Mello L, Meldrum B (1986) 2-Amino-7-phosphonoheptanoic acid (2-APH) infusion into entopeduncular nucleus protects against limbic seizures in rats. Neurosci Lett 64:226-230.

Paxinos G, Watson C (1997) The rat brain in stereotaxic coordinates. New York: Academic.

Pellegrino LJ, Pellegrino AS, Cushman AJ (1973) A stereotaxic atlas of the rat brain. Meredith, NY: Plenum.

Piredda S, Gale K (1985) Evidence that the deep prepiriform cortex contains a crucial epileptogenic site. Nature 317:623-625.

Price Jr RH, Hollingsworth Z, Young AB, Penney Jr JB (1993) Excitatory amino acid receptor regulation after subthalamic nucleus lesions in the rat. Brain Res 602:157-160.

Proctor M, Gale K (1998) Basal ganglia and brainstem anatomy and physiology. In: Epilepsy: a comprehensive textbook (Engel Jr J, Pedley TA, eds), pp353-368. Philadelphia: Lippincott-Raven.

Rinvik E, Otterson OP (1993) Terminals of subthalamonigral fibers are enriched with glutamate-like immunoreactivity: an electron microscopic, immunogold analysis in the cat. J Chem Neuroanat 6:19-30.

Robledo P, Feger J (1990) Excitatory influence of rat subthalamic nucleus to substantia nigra pars reticulata and the pallidal complex: electrophysiological data. Brain Res 518:47-54.

Scheel-Kruger J (1986) Dopamine-GABA interactions: evidence that GABA transmits and mediates dopaminergic functions in the basal ganglia and limbic system. Acta Neurol Scand Suppl 73:1-50.

Scheel-Kruger J, Arnt J, Magelund G (1977) Behavioral stimulation induced by muscimol and other GABA agonists injected into the substantia nigra. Neurosci Lett 4:351-356.

Scheel-Kruger J, Magelund G (1981) GABA in the entopeduncular nucleus and the subthalamic nucleus participates in mediating dopaminergic striatal output functions. Life Sci 29:1555-1562.

Sellal F, Hirsch E, Lisovoski F, Mutschler V, Collard M, Marescaux C (1992) Contralateral disappearance of parkinsonian signs after subthalamic hematoma. Neurology 42:255-256.

Sperber EF, Wurpel JND, Sharpless NS, Moshe SL (1989) Intranigral drug effects on striatal dopamine activity. Pharmacol Biochem Behav 32:1067-1070.

St-Pierre JA, Bedard PJ (1994) Intranigral but not intrastriatal microinjection of the NMDA antagonist MK-801 induces contralateral circling in the 6-OHDA rat model. Brain Res 660:255-260.

Takada M, Nishihama MS, Nishihama CC, Hattori T (1988) Two separate neuronal populations of the rat subthalamic nucleus project to the basal ganglia and pedunculopontine tegmental region. Brain Res 442:72-80.

Thach WT (1999) Fundamentals of motor systems. In: Fundamental neuroscience (Zigmond MJ, Bloom FE, Landis SC, Roberts JL, Squire LR, eds), pp 855-861. San Diego: Academic.

Turski L, Cavalheiro A, Turski A, Meldrum BS (1986) Excitatory neurotransmission within substantia nigra pars reticulata regulates the threshold for seizures produced by pilocarpine in rats: effects of intranigral 2-amino7-phosphonoheptanoate and $N$-methyl-D-aspartate. Neuroscience 18:61-77.

Veliskova J, Velsek L, Moshe SL (1996) Subthalamic nucleus: a new anticonvulsant site in the brain. NeuroReport 7:1786-1788.

Waddington JL (1978a) Rotational behavior and striatal dopamine metabolism following unilateral activation of nigral GABA mechanisms: GABA-like modulation of dopaminergic and nondopaminergic neurons in rat substantia nigra. Br J Pharmacol 63:384P.

Waddington JL (1978b) Behavioral evidence for GABAergic activity of the benzodiazepine flurazepam. Eur J Pharmacol 51:417-422.

Whittier JR, Mettler FA (1949) Studies on the subthalamus of the rhesus monkey. II. Hyperkinesia and other physiologic effects of subthalamic lesions, with special reference to the subthalamic nucleus of Luys. J Comp Neurol 90:319-372. 